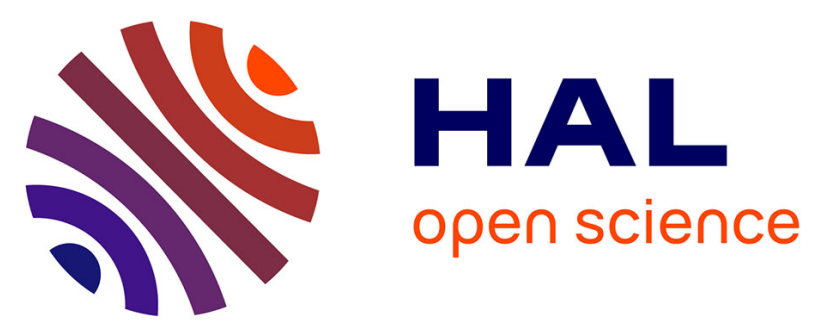

\title{
Agriculture sur pentes au Vietnam: une nécessité pour la sécurité alimentaire et un risque pour la durabilité du système agricole
}

Didier Orange, Pham Quang Ha, Toan Tran Duc, Floriane Clément, Pascal Jouquet, Phuong Nguyen Duy, Nguyen van Bo

\section{To cite this version:}

Didier Orange, Pham Quang Ha, Toan Tran Duc, Floriane Clément, Pascal Jouquet, et al.. Agriculture sur pentes au Vietnam: une nécessité pour la sécurité alimentaire et un risque pour la durabilité du système agricole. Roose Eric (ed.); Duchaufour H. (ed.); De Noni Georges (ed.). Lutte antiérosive: réhabilitation des sols tropicaux et protection contre les pluies exceptionnelles, IRD, 15 p., 2012, Colloques et Séminaires. ird-00712692

\section{HAL Id: ird-00712692 \\ https://hal.ird.fr/ird-00712692}

Submitted on 27 Jun 2012

HAL is a multi-disciplinary open access archive for the deposit and dissemination of scientific research documents, whether they are published or not. The documents may come from teaching and research institutions in France or abroad, or from public or private research centers.
L'archive ouverte pluridisciplinaire HAL, est destinée au dépôt et à la diffusion de documents scientifiques de niveau recherche, publiés ou non, émanant des établissements d'enseignement et de recherche français ou étrangers, des laboratoires publics ou privés. 
In : Roose Eric (ed.), Duchaufour H. (ed.), De Noni Georges (ed.), Lutte antiérosive : réhabilitation des sols tropicaux et protection contre les pluies exceptionnelles. Marseille : IRD, 2012

Orange Didier, Pham Quang H., Tran Duc T., Clément F., Jouquet P., Duy Phuong N., Van Bo Nguyen. Agriculture sur pentes au Vietnam : une nécessité pour la sécurité alimentaire et un risque pour la durabilité du système agricole. In : Roose Eric (ed.), Duchaufour H. (ed.), De Noni Georges (ed.), Lutte antiérosive : réhabilitation des sols tropicaux et protection contre les pluies exceptionnelles. Marseille : IRD, 2012, 15 p. (Colloques et Séminaires). ISBN 978-2-7099-1728-5

\title{
Agriculture sur pentes au Vietnam: une nécessité pour la sécurité alimentaire et un risque pour la durabilité du système agricole
}

\author{
Didier Orange ${ }^{(2)}$, Pham Quang Ha ${ }^{(1)}$, Tran Duc Toan ${ }^{(3)}$, Floriane Clément ${ }^{(2)}$, \\ Pascal Jouquet ${ }^{(2)}$, Nguyen Duy Phuong ${ }^{(3)}$ et Nguyen Van Bo ${ }^{(4)}$
}

(1) IAE, Institute for Agricultural Environment, VAAS, MARD, Hanoi; (2) IRD/IWMI, MSEC program, UMR211-Bioemco, Hanoi ; (3) SFRI, Soils and Fertilizers Research Institute, VAAS, MARD, Hanoi; (4) VAAS, Vietnamese Academy for Agricultural Science, MARD, Hanoi, Vietnam Correspondant et conférencier: Dr Pham Quang Ha, haphamquang@fpt.vn

\section{Résumé :}

Tandis que les zones de montagne moins peuplées apportent les ressources en eau qui permettent aux plaines de prospérer, les habitants des zones montagneuses continuent à souffrir de pauvreté, de faibles productivités agricoles accompagnées de dégradation environnementale. Une solution à ce paradigme semble être la mobilisation mutuelle de toutes parties prenantes via des mécanismes incitatifs de type PES (Payment for Environmental Services). L'idée est de relier politiques agricoles et stratégies individuelles dans une même action répondant aux contraintes physiques, économiques et culturelles des milieux physiques et humains en même temps qu'aux contraintes institutionnelles pour une bonne gouvernance. Un meilleur lien entre terres de pente et plaines devrait garantir un usage durable des terres de pente sans risque pour les plaines.

Mots clés: Vietnam, Montagnes, Système agricole, Actions incitatives, Gestion de Bassin versant, Asie du Sud-est

\begin{abstract}
:
While the mountainous area few populated provides the water resources to enrich the flat lands, the livelihoods of inhabitants in the mountainous area remain still poor, with weak agricultural productivity and environmental degradation. A solution to this paradigm could be the mutual involvement of each stakeholder by some incentive mechanism based on the sustainable development. The PES (Payment for Environmental Services) could then link agricultural policies with individual strategies to address both to the physical, economic and cultural constraints and to the institutional constraint for a best governance. One can expect by this way a better link between sloping lands and plains to guarantee a sustainable use of the sloping lands without risk for the flat lands.
\end{abstract}

Key words: Mountain, Farming system, Incentive Mechanisms, Watershed management, Southeast Asia 
In : Roose Eric (ed.), Duchaufour H. (ed.), De Noni Georges (ed.), Lutte antiérosive : réhabilitation des sols tropicaux et protection contre les pluies exceptionnelles. Marseille : IRD, 2012

\section{INTRODUCTION}

Sur les deux dernières décades, le Vietnam a connu sur tout son territoire d'énormes changements liés aux réformes agraires et à la croissance économique qui en a découlé (Le Trong Cuc, Rambo 2001 ; Castella J.C., Dang Dinh Quang, 2002 ; Castella et al., 2005 ; Tran Duc Vien, 2003). L'agriculture est passée d'un système agricole collectiviste géré par des coopératives à un système d'exploitations agricoles privées dirigées par des décisions individuelles. Le changement de la répartition des terres qui a suivi cette libéralisation économique s'est accompagné d'une importante augmentation de la productivité agricole et d'une diminution de la pauvreté (Le Trong Cuc, Rambo 2001). Cependant, l'amélioration n'a pas été uniforme (Tran Duc Vien, Rambo, 2001). Le développement économique dans les zones de pente (montagnes et collines) a été plus lent, laissant encore aujourd'hui des régions où la pauvreté est toujours présente, bien que le développement économique des terres de pente ait été souvent placé comme prioritaire dans les programmes de soutien des décideurs, bailleurs et organisations internationales non gouvernementales (ONG).

Or les terres de pente sont nécessaires pour le développement économique harmonieux du pays. En effet, le Vietnam compte aujourd'hui plus de 80 millions d'habitants et ne possède que 11 millions d'hectares de terres agricoles, faisant craindre la perte de capacité du pays à se nourrir, d'autant que le Vietnam est l'un des pays qui pourrait être le plus affecté par le changement climatique (Dasgupta et al., 2007). Les deux deltas (du Mékong et du Fleuve Rouge) qui constituent les zones majeures de production agricole du pays se transforment en zones industrielles et mégapoles. Dans le reste du pays, les plaines, dédiées initialement aux productions agricoles, sont de plus en plus fortement peuplées et industrialisées aux dépends des surfaces agricoles (Dasgupta et al., 2007). Dans le même temps, l'intensification et la densification de l'usage des terres de pentes a conduit à des impasses environnementales, malgré les programmes techniques de soutien aux politiques d'agriculture de conservation. D'une part, ces programmes avaient pour objectif d'améliorer ou de remplacer les systèmes supposés non adaptés des paysanneries traditionnelles. D'autre part, de nombreux programmes ont promus la reforestation pour la protection des sols et la gestion des bassins versants amont. Mais le succès de ces initiatives n'a jamais été celui escompté, aussi bien en ce qui concerne l'amélioration des conditions de vie que les bénéfices environnementaux (Sunderlin et Huynh Thu Ba, 2005 ; World Bank, 2008).

Ainsi tandis que les zones de montagne moins peuplées apportent les ressources en eau qui permettent aux plaines de prospérer, les habitants des zones montagneuses continuent à souffrir de pauvreté, de faibles productivités agricoles accompagnées de dégradation environnementale (Lang, 2001 ; Tran Duc Vien et Rambo, 2001 ; Tran Duc Toan et al., 2003 ; Valentin et al., 2008), qui en retour affecte de plus en plus les usages de l'eau et les populations des zones basses (Lundqvist, 2009). En effet, en dépit des efforts de soutien et des innovations technologiques, l'érosion continue à diminuer la fertilité des sols sur les terres de pente affectant le revenu des agriculteurs (Lestrelin et al., 2005 ; Valentin et al., 2008) et créant un risque écologique et économique pour les communautés d'en-bas du fait d'eaux polluées en nitrates et d'apports en sédiments qui comblent les retenues d'eau destinées à l'irrigation (Grandidier et 
Orange, 2008 ; Orange et al., 2008a ; Pham Quang Ha et Orange, 2008). Aussi un des grands défis actuels du Gouvernement Vietnamien est le développement de systèmes agro-écologiques économiquement rentables et durables qui permettront l'utilisation des terres de pentes par les petits agriculteurs (Orange et al., 2008b).

Par ailleurs, même si les technologies nouvelles peuvent être une solution technique efficace, il est reconnu depuis longtemps que les conditions d'adoption par les agriculteurs peuvent être un blocage important (Lestrelin et al., 2005; Noble et al., 2006; Clement et al., 2007 ; Clement et Amezaga, 2009) et nécessité des approches complexes, directes ou indirectes (Aldy et al., 1998 ; Haberl et al., 2004 ; Noble et al., 2006 ; Clement, 2008 ; Clement et Amezaga, 2008 ; Orange et al., 2008c ; Hayashi et al., 2009). En effet, une étude exhaustive de par le monde (de l'Afrique à l'Asie, des Amériques à l'Europe) des projets agricoles à succès a relevé que les facteurs clés de la réussite pouvaient se classer selon quatre catégories majeures: les contraintes individuelles, les contraintes sociales, les contraintes techniques et enfin les contraintes extérieures (Joshi et al., 2005 ; Noble et al., 2006).

Dans cet article, nous proposons une analyse des principaux requis pour promouvoir le développement agricole sur pente dans le Nord Vietnam afin de répondre à la fois aux contraintes de sécurité alimentaire et de réduction de la pauvreté d'une part, et aux contraintes de conservation des ressources en eau et en sol d'autre part. Notre discours est basé sur l'expérience de plusieurs projets de recherche menés au sein du Soils and Fertilizers Research Institute (SFRI) avec le programme international de recherche MSEC (Management of Soil Erosion Consortium) géré en collaboration entre I'IWMI et I'IRD (Maglinao et al., 2001 ; Valentin et al., 2008), notamment à partir d'un suivi à long terme de l'évolution des pratiques agricoles dans les zones montagneuses du Nord Vietnam basé sur des mesures hydrologiques et de pertes en sol par érosion couplées à des actions agricoles participatives. De ces expériences, il sera introduit la notion d'approches incitatives pour lier politiques agricoles et stratégies individuelles.

\section{AGRICULTURE SUR PENTE : LES CONTRAINTES}

Dans le Nord Vietnam, les collines et petites montagnes ont été déforestées dans les années 1970 pour l'usage agricole ; cela a eu pour conséquence un rapide appauvrissement des sols liés surtout aux pertes de nutriments par lessivage et induisant des changements des caractéristiques physiques et biologiques des sols. La situation actuelle des pentes est souvent dramatique, ne permettant plus que la culture de manioc avant d'aboutir à la jachère ou forêt.

Les activités de recherche de MSEC au Vietnam ont notamment concerné le suivi journalier à long terme (de 1999 à nos jours) des exportations de matières par les eaux d'écoulement de surface à l'échelle de petits bassins versants agricoles sur pente (de 1 ha à 50 ha) (Tran Duc Toan et al., 2003a, b ; Phan Ha Hai An et al., 2008 ; Orange et al., 2008b). Dans le bassin d'étude, les terres cultivées ont des pentes de $40 \%$ à plus de $100 \%$. Le couvert végétal majeur est passé 
progressivement de la culture de manioc sur tous les versants (40\% du couvert en 2001 à moins de $0,5 \%$ en 2004) à la plantation d'arbres pour pate à papier (Acacia mangium) et de fourrage tropical (Bracharia ruzziziensis) (Photo 1). Après 5 ans de suivi continu, les taux d'érosion mesurés sur des versants de 5 à 10 ha, ont confirmé que le nettoyage des sols (défrichage, labour, sarclage) générait les plus fortes pertes en terre (pouvant être supérieures à $10 \mathrm{t} / \mathrm{ha} / \mathrm{an}$ ) et que la forêt ou le couvert pluriannuel, tel le fourrage, éliminait toute érosion dès la deuxième année pour après 3-4 ans réduire l'érosion à moins de 0,5 t/ha/an (fig. 1).
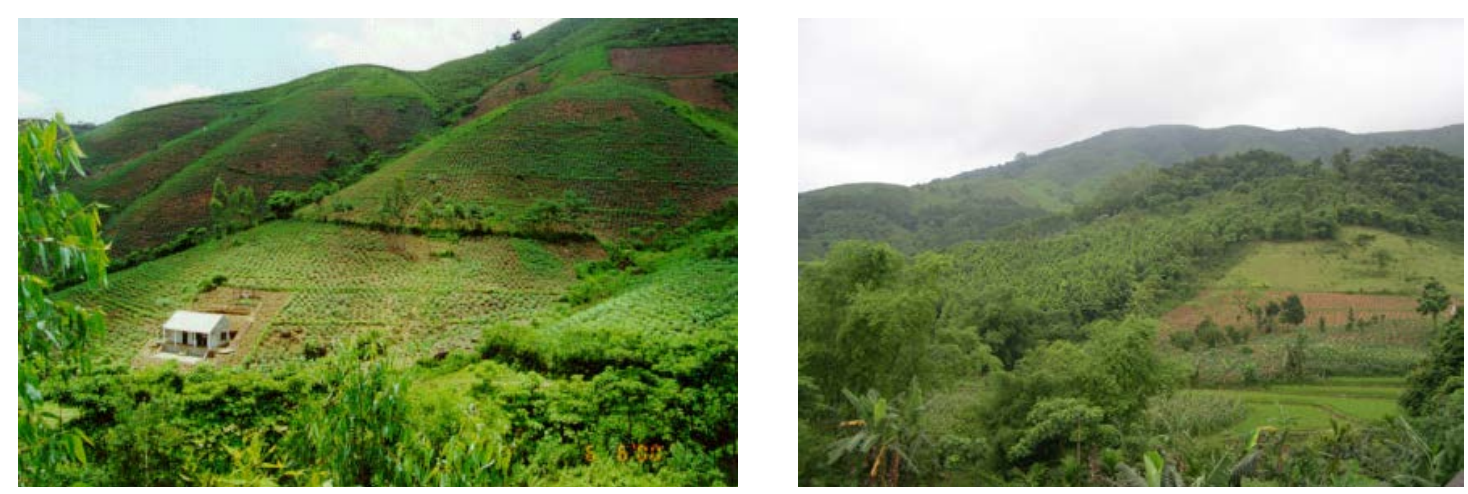

Photo 1: Illustration du changement d'usage agricole entre 2001 et 2005 dans la zone d'étude du programme MSEC (basin versant de Dong Cao, province de Hoa Binh, Nord Vietnam).copyright@ : D. Orange

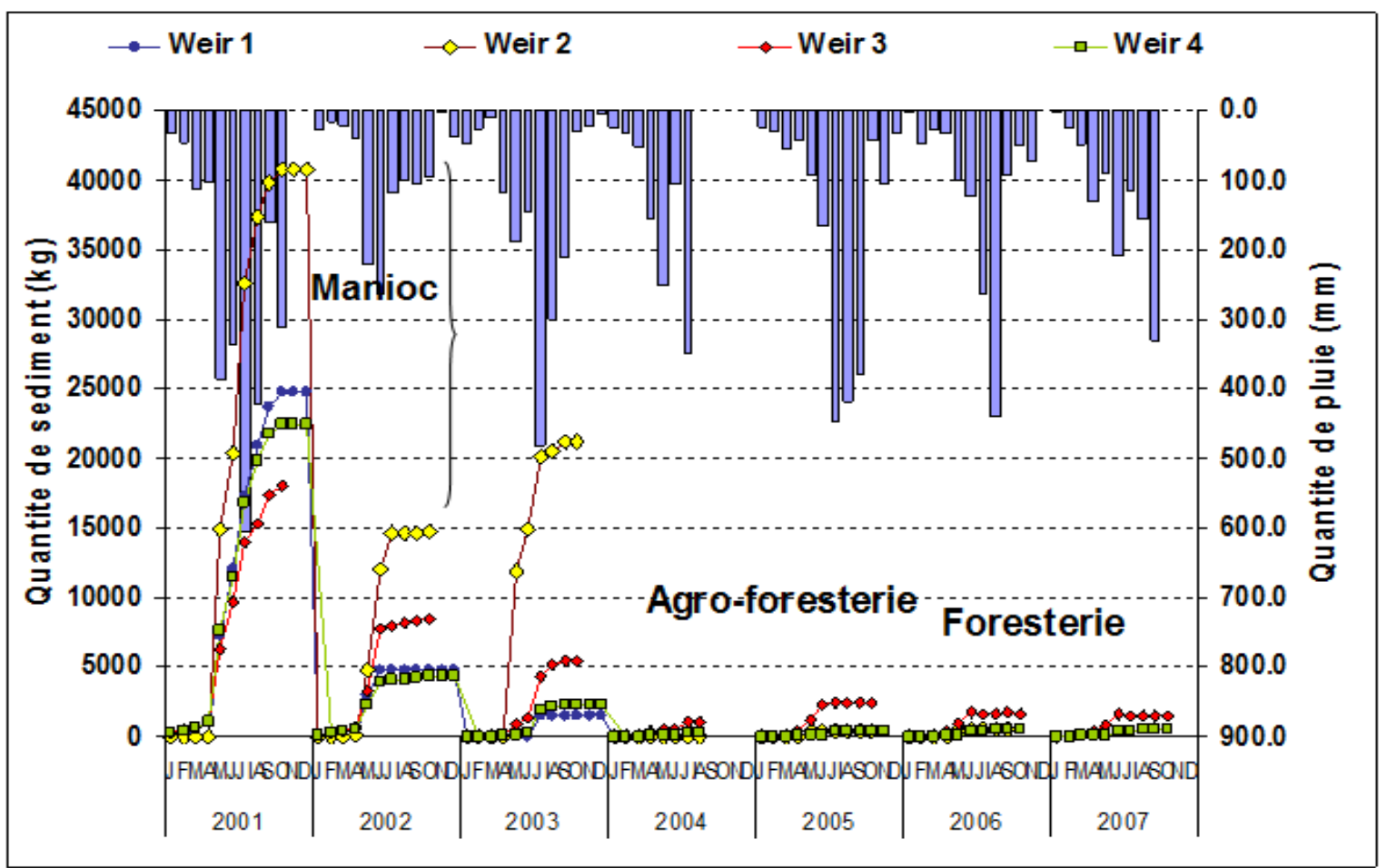

Fig. 1: Influence de l'utilisation des terres sur l'érosion. Evolution de 2001 à 2007 de la pluie mensuelle (en histogramme) et des courbes de cumul mensuel des pertes en terre de fond (bedload) mesurées sur 4 sous-bassins de 5 à 10 ha (Dong Cao Village, Hoa Binh Province, Northern Vietnam). Exutoires: Weir 1 (forêt), Weir 2 (plantation interannuelle, fourrage de Bracharia à partir de 2003), Weir 3 (manioc et agroforesterie à partir de 2003), Weir 4 (vieille jachère $>10$ ans) . 
In : Roose Eric (ed.), Duchaufour H. (ed.), De Noni Georges (ed.), Lutte antiérosive : réhabilitation des sols tropicaux et protection contre les pluies exceptionnelles. Marseille : IRD, 2012

Cependant dans le détail (Podwojewski et al., 2008), nous avons montré que ces valeurs d'érosion étaient directement liées à la capacité d'infiltration due aux modalités d'usage du sol. La comparaison de ces résultats avec les autres bassins versants expérimentaux MSEC (réseau de mesures réparti sur 5 pays d'Asie du Sud-est) confirme que l'érosion est effectivement plus expliquée par l'absence de couvert végétal que par son existence (Valentin et al., 2008). Ainsi, on confirme que la pratique agricole sur pente à une influence directe sur l'exportation de matière à l'exutoire de petits bassins versants agricoles inférieurs à $1 \mathrm{~km}^{2}$. Et on démontre que le meilleur indicateur de ces pertes en terre est le pourcentage de culture de maïs surtout, et en général de cultures annuelles, et non le pourcentage de forêt ou encore de couvert végétal.

Le suivi des pratiques culturales mené auprès des villageois de Dong Cao montre que les stratégies paysannes sont avant tout opportunistes, très liées aux lois du marché. Ainsi on voit depuis 2008 une recrudescence des cultures de maïs du fait de la demande économique tendue par le marché international. Même si l'agriculteur a conscience de la baisse de fertilité des sols de pente après des cycles de cultures annuelles (e.g. les rendements de manioc sont passés de 20 t/ha/an à moins de 10 t/ha/an en moins de 10 ans) et des risques de pertes en terre par érosion, ces deux contraintes n'influencent pas en priorité sa prise de décision (Clément et al., 2007).

\section{EXEMPLE D'IMPACT D'UNE POLITIQUE AGRICOLE : LA REFORESTATION}

Dans beaucoup de pays du monde, la réponse politique à une telle situation a été l'aménagement d'une politique volontaire de reforestation dans les zones montagneuses, i.e. le programme 5MHRP au Vietnam (MARD \& 5MHRP Partnership Secretariat, 2001). Le projet MSEC s'est intéressé à mesurer l'impact d'une telle politique à l'échelle d'une grande région du Nord Vietnam (i.e. la Province de Hoa Binh) et au niveau individuel (Clement, 2008 ; Clement et al., 2007, 2009). Pour cela, une méthodologie originale d'analyse a été utilisée: Institutional Analysis and Development (IAD) largement documentée dans Clément (2008). Cette méthode permet de mettre en relief l'analyse du changement d'usage en prenant en compte à la fois les aspects institutionnels avec une perspective historique et les aspects liés à la perception des acteurs et leur discours concernant la gestion des terres et la forêt (fig. 2).

Les résultats montrent que les politiques nationales ont interféré fortement avec les facteurs locaux, conduisant localement à une compétition complexe entre la prise de décision et l'action (Clement et Amezaga, 2008, 2009). Ainsi la plantation d'arbres n'est pas une réponse des agriculteurs à la politique incitative de reforestation mais plutôt un résultat non attendu d'un dysfonctionnement des institutions locales vis-à-vis de cette politique du fait d'une exagération des croyances et discours non fondés de la part des commanditaires nationaux. Cette déformation de la réalité a engendré un conflit de perception conduisant à des résultats non prévisibles et localement dépendants. 


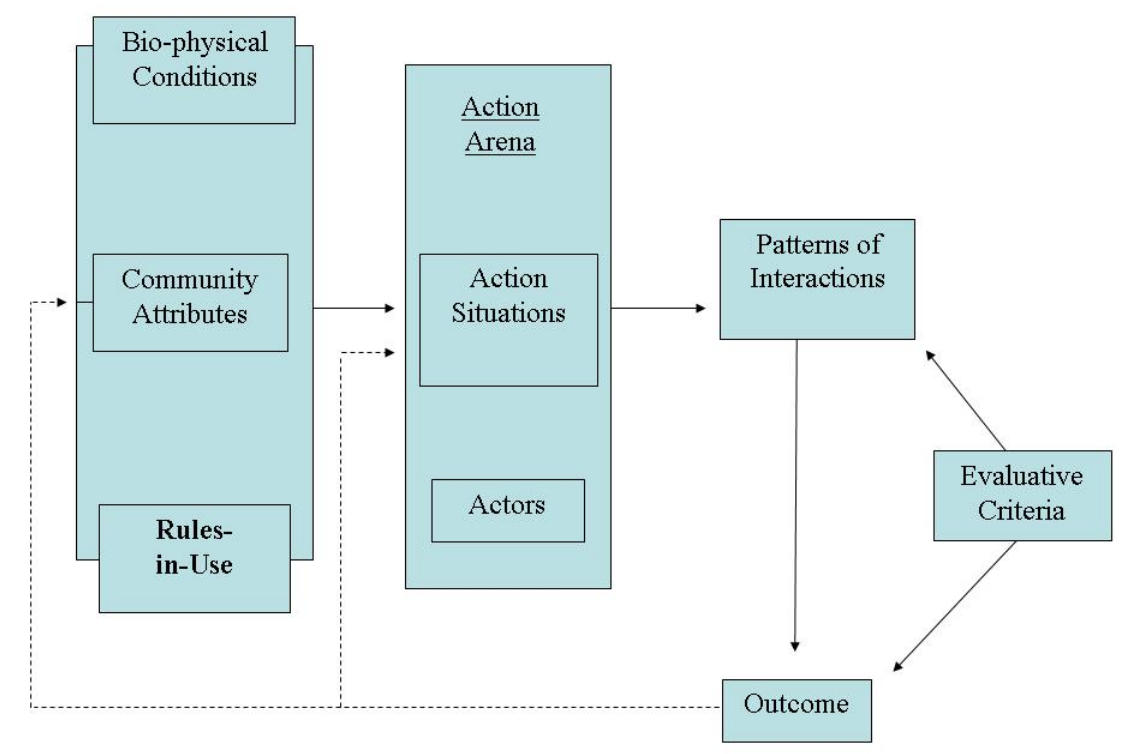

Fig. 2: Schéma d'analyse de la méthode IAD (Institutional Analysis and Development). Source: Kiser and Ostrom, 1982; E. Ostrom et al., 1994

Une étude de la dynamique spatiale de la reforestation dans la Province de Hoa Binh par une méthode statistique spatiale croisée avec une imagerie satellitale confirme cette analyse (Clement et al., 2009). II apparait que les facteurs ayant eu le plus d'impact sur la reforestation sont d'abord la proximité d'industries du bois, la distance à la grande route, puis seulement l'allocation des terres aux agriculteurs (Clement et al., 2009). Par ailleurs, la comparaison des valeurs de couvert végétal dans la Province de Hoa Binh entre 1993 et 2000 indiquent qu'on doit être extrêmement prudents quant à l'utilisation de ces données. Sur la base de l'imagerie satellitale, nos estimations sont deux à dix fois moins fortes que les chiffres délivrés par le gouvernement. La question de l'extension de la forêt est donc bien à remettre en cause. Pourquoi une telle déformation?

Nos résultats tendent à montrer que le programme 5MHRP a été mal adapté aux conditions socio-écologiques du Nord Vietnam, ne permettant pas alors l'implication des agriculteurs locaux dans la dynamique finale du projet qui consistaient à l'obtention d'une allocation à long terme de la terre à la forêt. Les agriculteurs ont effectivement planté la forêt comme une opportunité au remplacement d'une culture annuelle devenue non rentable sur des sols appauvris, mais cette forêt a été coupée après 3 à 6 ans sans continuation obligatoire. Aussi ce décalage entre l'action et la demande nous a conduit aux recommandations suivantes (Clement et al., 2009): (1) l'augmentation de la responsabilité de l'administration vis-à-vis du gouvernement et des populations ; (2) l'amélioration de la formation des décideurs quant à la diversité et la complexité des conditions socio-écologiques d'un milieu naturel; et (3) l'adaptation des institutions concernées à cette complexité pour la gestion des terres et de la forêt par l'attribution d'une plus grande responsabilité aux acteurs locaux. 
In : Roose Eric (ed.), Duchaufour H. (ed.), De Noni Georges (ed.), Lutte antiérosive : réhabilitation des sols tropicaux et protection contre les pluies exceptionnelles. Marseille : IRD, 2012

\section{STRATEGIES INDIVIDUELLES : LECONS D'UNE APPROCHE OPPORTUNISTE DANS LE VILLAGE DE DONG CAO}

Revenons dans le village expérimental de Dong Cao. En 2001, toutes les pentes étaient cultivées en manioc (photo 1). En 2004-2006, la majorité des pentes de la zone étaient plantées en forêt avec des parties de fourrage. A partir de 2007, les fourrages disparaissent, les forêts sont coupées sans être remplacées, on retrouve le manioc et le maïs qui dominent à nouveau le paysage. Dans les exploitations agricoles, au cours de la même période, l'élevage (surtout porcin, un peu bovin) s'est développé. Le paysage s'est clôturé et les plus riches ont investi dans un bio-digesteur destiné à la production de gaz de cuisine.

En 2003, l'intérêt de la culture du fourrage sur pente comme une bonne alternative aux cultures annuelles (telles que le manioc et le maïs) pour le contrôle de l'érosion sur pente a conduit les chercheurs à promouvoir l'intégration de l'élevage dans les systèmes d'exploitation agricole. Dans le même temps, la réussite économique du développement de la filière porcine dans tout le Vietnam depuis la fin des années 90 a stimulé l'écoute des agriculteurs quant à l'intérêt du fourrage. Ils ont vu là un avantage direct pour un retour sur investissement plus rapide et plus important que la reforestation. Sur cette base, le projet DurasCropLivestock se développait sous la responsabilité du SFRI pour accompagner la demande des agriculteurs dans le développement de l'intégration de l'élevage et la gestion de la fertilité des sols (Orange et al., 2008c). Le projet MSEC a commencé en 2004 la culture de fourrage d'espèces locales sur pente avec 5 agriculteurs dans un village. En 2006, le projet Duras démarrait en concertation entre la demande scientifique des chercheurs et la demande de développement des agriculteurs assistés par les agents techniques et scientifiques du SFRI (du ministère de l'Agriculture Vietnamien). En 2007, on comptait 300 agriculteurs qui pratiquaient la culture de fourrage répartis sur 7 communes (i.e. plus de 50 villages). Une évaluation vient d'être faite en 2009 (en cours de rédaction), il n'y a pratiquement plus de cultures de fourrage. Cette enquête récente confirme ce que nous écrivions fin 2007 dans les conclusions du projet Duras : "The impact on the erosion was immediate. Afforestation and the fodder crops stopped soil losses on sloping lands within the studied area. However, a wish to decrease soil erosion was not the driving force that led to the adoption of these more conserving approaches by farmers. Improved incomes associated with forestry and fodder production (i.e. an additional farming element in the form of livestock production) were the main reasons for adoption".

L'engagement initial des agriculteurs et des agents vulgarisateurs durant la phase de montage du projet a été un pré-requis important à la diffusion de la technique fourragère via leur implication directe dans l'établissement de champs de démonstration prêtés sur les zones communales des villages. Par ailleurs, la nature des essais (type de techniques culturales, type d'espèces fourragères, calendrier de fertilisation...) était entièrement déterminée à partir de rencontres agriculteurs, décideurs locaux et chercheurs. La principale idée était de laisser libre l'initiative locale. Ainsi l'expérimentation de fourrages tempérés (de type avoine) sur les champs de riz laissés libres l'hiver fut une proposition des 
agriculteurs afin de répondre à l'une de leur contrainte majeure qui est de pouvoir nourrir leurs animaux durant l'hiver. En effet, dans le Nord Vietnam l'hiver peut être rude, avec des températures inférieures à $10^{\circ} \mathrm{C}$ et donc une productivité végétale des espèces tropicales quasi nulle. Après 1 an, $80 \%$ des agriculteurs des 3 villages tests cultivaient du fourrage dans leur champ de riz durant l'hiver. C'est aujourd'hui la seule culture de fourrage qui reste encore un peu, la désaffection de cette pratique est liée à la non production des graines d'avoine par les services compétents de l'Etat. II y a eu donc cette fois un manque d'appropriation du côté des services gouvernementaux.

En effet, si la diversité des institutions impliquées dans ce projet (de la recherche au village en passant par les services agricoles de l'Etat) a assuré de répondre à la diversité des problèmes liés au développement agricole, un autre facteur qui doit aussi être pris en compte, est la durée du projet (ici, deux ans seulement !). Le manque de temps crée non seulement un manque d'occasion de partages mais aussi une fatigue latente de l'agriculteur vis-à-vis du message continu des services agricoles concernant le besoin de développement.

Enfin, il faut également retenir de cette expérience l'opportunisme de la part des chercheurs, et non seulement des agriculteurs, pour développer une collaboration (i.e. le projet Duras-CropLivestock) qui a permis le développement de leurs essais agricoles pour l'amélioration de leurs modèles (Jouquet et al., 2007 ; Valentin et al., 2008).

\section{DISCUSSION : MAITRISE TECHNIQUE OU ATTENTE SOCIALE ? APPROCHES INCITATIVES}

De tout cela, il faut retenir l'opportunisme de situation et le pragmatisme qui peut conduire d'une part à des changements d'usage rapides et spectaculaires de la part des agriculteurs, et d'autre part à des collaborations fructueuses entre agriculteurs et scientifiques, chacun y trouvant ce qu'il recherche. Les changements sont donc possibles, encore faut-il en trouver la voie?

Cette voie interroge à la fois l'idée du développement durable (chacun ayant sa propre représentation) et les notions d'efficacité et de stratégie fortement liées aux contraintes culturelles qui pèsent sur chaque individu (Jullien, 2005). Ce n'est pas le lieu d'en discuter ici. On retiendra simplement que le déterminisme technologique posé comme d'une part «naturellement bon » et d'autre part capable de créer un «monde meilleur », est passé (Robin J., 1989 ; Roco et Bainbridge, 2002 ; Heisbourg, 2007). Au lieu de se focaliser sur des finalités technologiques, il convient de placer sur un même plan les défis sociaux et écologiques comme moteur de la réflexion et les technologies comme moyen. La méthode IAD évoquée en début de cet article permet justement de considérer la participation sociale pour orienter les priorités techniques. En bref, ce n'est pas la société qui s'adapte aux avancées techniques mais l'inverse. Sur ce sujet, il est remarquable de constater que l'analyse statistique croisée des résultats du programme MSEC sur 27 bassins versants expérimentaux répartis entre I'Indonésie, la Laos, les Philippines, le Laos et le Vietnam montre que l'adoption 
des technologies de gestion des sols de pente n'est pas fonction du degré d'intensification des systèmes d'exploitation ni du niveau des revenus (Valentin et al., 2008).

Le maitre mot est donc bien l'attente sociale et non la maitrise technique (Jasanoff, 2007 ; Clement et al., 2009). Cependant, il reste à trouver la bonne « formulation » pour motiver les différents dépositaires.

Aussi au lieu de construire l'intervention du projet de développement sur une distinction entre connaissances locales et connaissances scientifiques, qui est en fait une construction abstraite souvent diffusée par la communauté scientifique (Forsyth, 1996), notre hypothèse de travail fut que les différents intervenants (de l'amont et de l'aval, et les décideurs) promeuvent -- aient pour volonté -- la durabilité des fonctions écosystémiques de leur région, se traduisant concrètement en terme de rentabilité des terres et des eaux (« gagner plus ? »), d'accès à la modernité (électricité, chauffage,...) et à la santé (« vivre mieux »). C'est dans cette optique que chercheurs, décideurs et agriculteurs se sont retrouvés à promouvoir ensemble, chacun dans sa sphère de compétence et d'intérêt, un même moyen technologique que représente le bio-digesteur (Orange et al., 2008c ; projet AFD Biogas\&PES, Hanio, Vietnam).

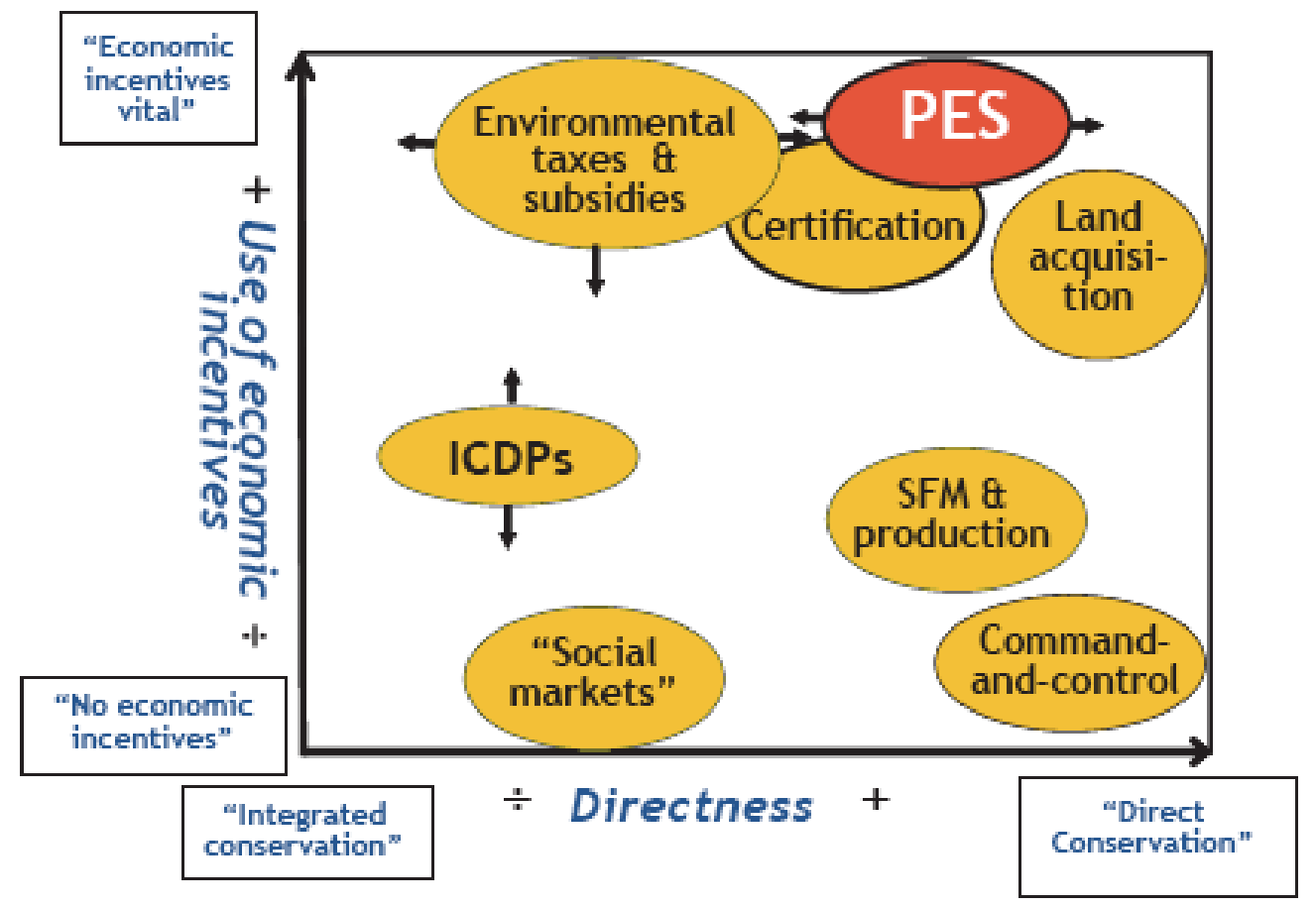

Fig. 3: Comparaison des systèmes PES (Payment for Environmental Services) avec les autres approches de conservation. Cette figure range les approches de conservation selon deux critères : (1) le degré du lien avec une incitation économique, (2) le degré d'importance apporté à la notion de conservation (ICDP : Integrated conservation and development projects ; SFM : Sustainable Forest management).

Source: Wunder, 2005

L'idée a été de promouvoir la technologie du biodigester via le concept de PES. A la suite du rapport Bruntland (1987) et de la Conférence de Rio (1992), les 
programmes de conservation des sols et des eaux en monde tropical sont de plus en plus orientés vers la prise en considération des personnes, considérant que l'éradication de la pauvreté était l'unique voie pour conserver et protéger l'environnement. Les incitations économiques, comme moyen à la fois de conservation de l'environnement et d'apport de revenus additionnels aux pauvres, sont au cœur de cette nouvelle approche pour le développement. Dans ce cadre, le concept de PES, Payment for Environmental Services, a été défini par Wunder (2005) selon les 5 critères suivants: (1) une transaction volontaire où (2) un service environnemental bien défini est (3) acheté par un acheteur (4) fourni par un fournisseur (5) si et seulement si the fournisseur du service environnemental garantit la durabilité de ce service. Le concept de PES cumule donc à la fois la notion d'incitation économique et la notion de volontariat partagé entre deux parties prenantes. II se situe entre un système directif d'attribution des terres ayant pour but d'éliminer les usages problématiques, i.e. les politiques dirigistes de reforestation par expropriation, et les systèmes à taxes et subventions sur base environnementale (fig. 3). La différence est que le PES essaye de mobiliser les acteurs plus sur la notion de conservation que sur celle de production économique à partir de l'usage d'une ressource.

Le montage imaginé et actuellement en préparation est représenté en figure 4. On voit que la structure permet de faire un lien entre des décideurs de même niveau (agriculteurs et décideurs ont égale importance), la structure étant renseignée par un « mécanisme de supervision » qui produit de l'information aussi bien technique que sociale et économique (i.e. gouvernance). Cela rappelle les systèmes mis en place pour les activités de micro-crédits (Yunus, 1997).

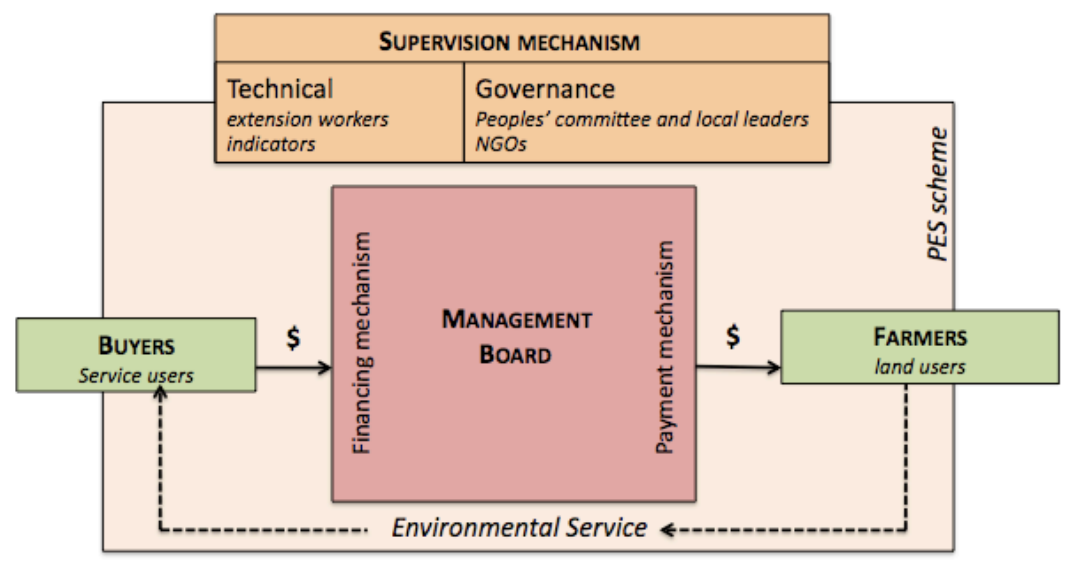

Fig. 4: Proposition d'un schéma d'un système de politique agricole basé sur un système PES.

\section{CONCLUSION}

Les exemples discutés dans ce papier ont souligné que l'impact des politiques agricoles dépendait non seulement de la politique elle-même et des facteurs macro et/ou méso économiques mais aussi des conditions locales sociales et biophysiques. Bien sûr, d'autres auteurs ont déjà mentionné ce problème, dans des cas aussi spécifiques que l'étude de l'érosion (e.g. Lal, 1983 ; 
Valentin et al., 2008), ou encore que l'étude de la fertilité des sols et de la gestion des fertilisants (e.g. Pierce and Larson, 1993 ; Doran, 2002 ; Van Keulen, 1995). Mais ce papier a aussi souligné que l'opportunisme d'action n'était pas seulement du fait de l'agriculteur mais aussi du chercheur et du décideur. II y a bien partage complet des responsabilités (Yunus, 1997 ; Sanders et al., 1999 ; Orange et al., 2002 ; Clement et al., 2009).

On a vu que les raisons qui font que les agriculteurs s'investissent sont liées à la faculté d'appropriation et de partage de l'information entre toutes les sphères de partenariat. En effet, chacun doit répondre à son propre intérêt : une valorisation économique et un libre choix pour l'agriculteur, une meilleure compréhension et diffusion des technologies agricoles sur le terrain pour le technicien agricole et le décideur, des résultats scientifiques probant en terme de gestion de la ressource pour le chercheur.

Finalement, la notion de durabilité environnementale est peut-être la seule notion qui puisse réunir un intérêt mutuel commun. Le nouveau concept de PES (Payment for Environmental Services) a été construit sur cette base (Pagiola et Platais, 2004 ; Tomich et al., 2004). Le challenge de cette approche est alors de mixer intérêt économique et engagement mutuel (George et al., 2009). Par ce biais, le concept permet d'assurer l'adaptation des politiques agricoles aux contraintes et surtout aux demandes locales. L'idée est de relier politiques agricoles et stratégies individuelles dans une même action répondant à la fois aux contraintes physiques, économiques et culturelles des milieux physiques et humains en même temps qu'aux contraintes institutionnelles et de gouvernance. Mais au-delà de la notion abstraite de développement durable (George et al., 2009), il semble nécessaire de pouvoir renseigner l'attente sociale via un objet technologique. Dans le cas du Nord Vietnam où les conditions de développement exigent une mise en agriculture des terres sur les pentes, le bio-digesteur comme objet d'intérêt mutuel entre agriculteurs et décideurs pourrait être une des solutions pour garantir une agriculture durable sur les terres de pente sans risque pour les terres de plaine.

\section{Remerciements}

Cette action de recherche est réalisée dans le cadre du programme MSEC de l'IWMI coordonnée par l'unité de recherche IRD-UMR211 (BIOEMCO) de Christian Valentin. Nous devons remercier les nombreux bailleurs qui ont permis le développement de ce programme pluridisciplinaire. On citera dans l'ordre chronologique le projet Duras-Croplivestock du MAE-GFAR, le projet PES du Challenge Program for Water and Food (contrat C-056-07), le projet Biogas\&PES de l'Agence locale AFD de Hanoi.

\section{BIBLIOGRAPHIE}

Aldy J.E., Hrubovcak J., Vasavada U., 1998. The role of technology in sustaining agriculture and the environment. Ecological Economics, Elsevier, 26 : 81-96.

Castella J.C., Boissau S., Trung T.N., Quang D.D., 2005. Agrarian transition and lowland-upland interactions in mountain areas in northern Vietnam: application of a multi-agent simulation model. Agricultural Systems, 86 (3): 312-332. 
In : Roose Eric (ed.), Duchaufour H. (ed.), De Noni Georges (ed.), Lutte antiérosive : réhabilitation des sols tropicaux et protection contre les pluies exceptionnelles. Marseille : IRD, 2012

Castella J.C., Dang Dinh Quang (Eds.), 2002. Doi Moi in the Mountains. Land Use Changes and Farmers' Livelihood Strategies in Bac Kan Province, Viet Nam. The Agricultural Publishing House, Hanoi: 480 p.

Clément F., 2008. A multi-level analysis of forest policies in Northern Vietnam: Uplands, people, institutions and discourses. MSEC programme, IWMI-IRD-SFRI consortium, Hanoi, Vietnam; PhD, Faculty of Science, Agriculture and Engineering, Newcastle University, July 2008: 263 p.

Clément F., Amezaga J.M., 2008. Linking reforestation policies with land use change in Northern Vietnam: Why local factors matter. Geoforum, Elsevier, 39(1): 265-277.

Clement F., Amezaga J.M.. 2009. Afforestation and Forestry Land Allocation in Northern Vietnam: Analysing the gap between policy intentions and outcomes. Land Use Policy, 26 (2):458-470.

Clement F., Amezaga J.M., Orange D., Tran Duc Toan, 2007. The Impact of Government Policies on Land Use in Northern Vietnam: An institutional approach for understanding farmer decisions. IWMI Research Report 112. Colombo, Sri Lanka: [online] URL: http://wwww.iwmi.cgiar.org/Publications/IWMI_Research_Reports/PDF/PUB112/RR112.pdf.

Clement, F., Orange D., Williams M., Mulley C., Epprecht M., 2009. Drivers of Afforestation in Northern Vietnam: Assessing local variations using geographically weighted regression. Applied Geography, doi:10.1016/j.apgeog.2009.01.003.

Dasgupta S., Laplante B., Meisner C., Wheeler D., Jianping Yan, 2007. The Impact of Sea Level Rise on Developing Countries: A Comparative Analysis. World Bank Policy Research Working Paper, 4136, February 2007, 51 p.

Doran, J.W., 2002. Soil health and global sustainability: translating science into practice. Agric. Ecosyst. Environ. 88, 119-127.

Haberl H., Wackernagel M., Wrbka T., 2004. Land use and sustainability indicators. An introduction. Land Use Policy, Elsevier, 21: 193 - 198.

Forsyth, T., 1996. Science, Myth and Knowledge: Testing Himalayan environmental degradation in Thailand. Geoforum 27, 375-392.

Hayashi K., Abdoulaye T., Matsunaga R., Tobita S., 2009. Appraisal of Local Farmers' Practices on Land Management for a Guideline of Agricultural Development in the Sahel Zone of Niger, West Africa. Japan Agricultural Research Quarterly, 43(1): 63-69.

Heisbourg F., 2007. L'épaisseur du Monde. Ed. Stock, Paris, 227 p.

Grandidier E., Orange D., 2008. Socio-economic and environmental assessments for PES implementation in a watershed in Northern Vietnam. Non published report, CPWF-PES-Vietnam project, MSEC-Vietnam project, SFRI-IWMI-IRD, Hanoi : 128 p.

George A., Pierret A., Boonsaner A., Valentin C., Orange D., Planchon O., 2009. Potential and limitations of Payments for Environmental Services (PES) as a means to manage watershed services in mainland Southeast Asia. Int. Journal of Commons, 3(1) : 16-40.

Jasanoff S., 2007. Science and politics technologies of humility. Nature: 450.

Joshi P.K., Jha A.K., Wani S.P., Joshi L., Shiyani R.L., 2005. Meta-analysis to assess impact of watershed program and people's participation. Comprehensive Assessment Research, report 8, Colombo, Sri Lanka, IWMI.

Jouquet P., Bernard-Reversat F., Bottinelli, N., Orange, D., Rouland-Lefèvre C., Tran Duc Toan, Podwojewski P., 2007. Influence of change in land use and earthworm activities on carbon and nitrogen dynamics in a steepland ecosystem in Northern Vietnam. Biology and Fertility of Soils, 44(1): 69-77 
In : Roose Eric (ed.), Duchaufour H. (ed.), De Noni Georges (ed.), Lutte antiérosive : réhabilitation des sols tropicaux et protection contre les pluies exceptionnelles. Marseille : IRD, 2012

Jullien F., 2005. Traité de l'efficacité. Ed. Grasset, Paris, 234 p.

Kiser L., Ostrom E., 1982. The Three Worlds of Action: A metatheoretical synthesis of institutional approaches. In: Ostrom, E. (Ed.), Strategies of Political Inquiry. Sage, Beverly Hills (CA), pp. 179222.

Lal R., 1983. Soil erosion in the humid tropics with particular reference to agricultural land development and soil management. In: Hydrology of humid tropical regions with particular reference to the hydrological effects of agricultura and forestry practice, Proc. Hamburg symposium, IAHS Publ. 140 : 221-239;

Lang C., 2001. Deforestation in Vietnam, Laos, and Cambodia. In: Vajpeyi, D.K. (Ed.), Deforestation, Environment, and Sustainable Development: A Comparative Analysis, Praeger, London: 111-137.

Le Trong Cuc, Rambo A.T. (Eds.), 2001. Bright Peaks, Dark Valleys. A Comparative Analysis of Environmental and Social Conditions and Development Trends in Five Communities in Northern Vietnam's Northern Mountain Region. The National Political Publishing House, Hanoi : $350 \mathrm{p}$.

Lestrelin G., Giordano M., Keohavong B., 2005. When "conservation" leads to land degradation: Lessons from ban Lak Sip, Laos. Research Report, 91, Colombo, Sri lanka, IWMI, 25 p.

Lundqvist J., 2009. Water as a Human Resource. Encyclopedia of Inland Waters: 31-42.

Maglinao A.R., Penning de Vries, F., Bricquet J.-P., 2001. Consortium Approach to Soil Erosion Management: A potential key in sustaining upland development in Asia. International Conference on Sustaining Upland Development in Southeast Asia, Makati City, Philippines.

Noble A.D., Bossio D.A., Penning de Vries F.W.T., Pretty J., Thiyagarajan T.M., 2006. Intensifying agricultural sustainability: An analysis of impacts and drivers in the development of "Bright spots". Comprehensive Assessment Research Report, 13, IWMI, Colombo, Sri Lanka: 32 p.

Orange D., Arfi R., Kuper M., Morand P., Poncet Y. (éd.), 2002. Gestion intégrée des ressources naturelles en zones inondables tropicales. Actes du colloque Girnzit, Bamako, 20-23 juin 2000. Paris, IRD, coll. Colloques et séminaires : $1000 \mathrm{p}$.

Orange D., Jouquet P., Tran Duc Toan, Montresor A., 2008a. Enhancing the health and incomes of communities in Northern Vietnam through improving household animal production and waste management for a sustainable environment. Final Report, Total collaborative project, MSEC team, IRD-IWMI, SFRI, Hanoi, Vietnam, 28 p.

Orange D., Lequeux B., Henry des Tureaux T., Pham Van Rinh, Tran Duc Toan, 2008b. Charges de fond et suspensions transportées par les eaux d'écoulement dans un petit bassin versant agricole sur pentes dans le Nord Vietnam. In : Orange D., Roose E., Vermande P., Gastellu J.-P., Pham Quang $\mathrm{Ha}$ (Eds) : Gestion intégrée des eaux et des sols: ressources, aménagements et risques en milieux ruraux et urbains, Editions AUF et IRD, Hanoi, Actes des Premières Journées Scientifiques Inter-Réseaux de l'AUF, Hanoi, 6-9 novembre 2007, cd-rom : 6p. (Communication orale).

Orange D., Tran Duc Toan, Nguyen Duy Phuong, Nguyen Van Thiet, Salgado P., Clement F., Le Hoa Binh, 2008c. Different interests, common concerns and shared benefits. LEISA Magazine, 24(2): 12-13. + Chinese version.

Ostrom E., Gardner R., Walker J., 1994. Rules, Games \& Common-Pool Resources. The University of Michigan Press, Ann Arbor.

Pagiola S., Platais G., 2004. Payments for environmental services: From theory to practice. Washington DC: World Bank. 
In : Roose Eric (ed.), Duchaufour H. (ed.), De Noni Georges (ed.), Lutte antiérosive : réhabilitation des sols tropicaux et protection contre les pluies exceptionnelles. Marseille : IRD, 2012

Pham Quang Ha, Orange D., 2008. Réseau E-GCES-Vietnam : Erosion et Gestion conservatoire des eaux et des sols. Bilan d'activités 2008, projet AUF-ISTEM, SFRI, Hanoi, Vietnam, 2 p.

Phan Ha Hai An, Orange D., Huon S., Henry des Tureaux T., Pham Van Rinh, Tran Thi My Linh, Podwojewski P., 2008. Evolution des teneurs en carbone organique et azote dans les matières en suspension des eaux de surface d'un petit basin versant agricole sur pente dans le Nord Vietnam. In Orange D., Roose E., Vermande P., Gastellu J.-P., Pham Quang Ha (Eds) : Gestion intégrée des eaux et des sols : ressources, aménagements et risques en milieux ruraux et urbains, Editions AUF et IRD, Hanoi, Actes des Premières Journées Scientifiques Inter-Réseaux de I'AUF, Hanoi, 69 novembre 2007, cd-rom : 6p. (Communication orale).

Pierce F.J., Larson W.E., 1993. Developing criteria to evaluate sustainable land management. In: Kimble, J.M. (Ed.), Proceedings of the Eighth International Soil Management Workshop: Utilization of Soil Survey Information for Sustainable Land Use. USDA Soil Conservation Service, National Soil Survey Center, Lincoln, NE, USA : 7-14.

Podwojewski P., Orange D., Jouquet P., Valentin C., Nguyen Van T., Janeau J.L., Tran Duc T., 2008. Land-use impacts on surface runoff and soil detachment within agricultural sloping lands in Northern Vietnam. Catena, $74: 109-118$

Robin J., 1989. Changer d'ère. Ed. Seuil, Paris, 235 p.

Roco M.C., Bainbridge W.S., eds, 2002. Converging technologies for improving human performance: nanotechnology, biotechnology, information technology and cognitive science. Arlington (Virginie), National Science Foundation, (http://www.nsf.gov/crssprgm/nano/reports/nsfnnireports.jsp).

Sanders D., Huszars P., Sombatpanit S., Enters, T. (eds.), 1999. Incentives for soil conservation from theory to practice. World Association of Soil and Water Conservation/Oxford and IBH Publishing, New Delhi.

Sunderlin W.D., Huynh Thu Ba, 2005. Poverty Alleviation and Forests in Vietnam. Center for International Forestry Research (CIFOR), Bogor, Jakarta, Indonesia.

Tomich T.P., Thomas D.E., van Noordwijk M., 2004. Environmental services and land use change in Southeast Asia: from recognition to regulation or reward. Agriculture, Ecosystems and Environment 104(1) : 229-244.

Tran Duc Toan, Orange D., Podwojewski P., Do Dui Phai, Thai Phien, Maugin J., Pham Van Rinh, 2003. Soil erosion and land use in the Dong Cao Catchment in Northern Vietnam. In: Maglinao A.R., Valentin C., Penning de Vries F. (Eds.), From soil research to land and water management: harmonizing people and nature. Proceedings of IWMI-ADB Project Annual Meeting and $7^{\text {th }}$ MSEC Assembly. IWMI. Southeast Asian Regional Office, Vientiane, Laos.

Tran Duc Toan, Orange D., Podwojewski P., Do Duy Phai, Thai Phien, 2003. Erosion control within a cultivated sloping land in North Vietnam. China Symposium 2 / Soil quality and evolution mechanism and sustainable use of soil resources, ISSAS / Yingtan, Jiangxi Province, China, September 23-28 2003.

Tran Duc Vien, 2003. Culture, Environment, and Farming Systems in Vietnam's Northern Mountain Region. Southeast Asian Studies 41: 180-205.

Tran Duc Vien, Rambo A.T., 2001. Social Organization. In: Le Trong Cuc, Rambo A.T. (Eds.), Bright Peaks, Dark Valleys. A Comparative Analysis of Environmental and Social Conditions and Development Trends in Five Communities in Northern Vietnam's Northern Mountain Region, The National Political Publishing House, Hanoi : 177-208.

Valentin C., Agus F., Alamban R., Boosaner A., Bricquet J.P., Chaplot V., de Guzman T., de Rouw A., Janeau J.L., Orange D., Phai Do Duy, Podwojewski P., Ribolzi O., Silvera N., Subagyono K., Thiébaux J.P., Toan Tran Duc, 2008. Runoff and sediment losses from 27 upland catchments in 
In : Roose Eric (ed.), Duchaufour H. (ed.), De Noni Georges (ed.), Lutte antiérosive : réhabilitation des sols tropicaux et protection contre les pluies exceptionnelles. Marseille : IRD, 2012

Southeast Asia: Impact of rapid land use changes and conservation practices. Agriculture, Ecosystems and Environment, 128: 225-238.

Van Keulen H., 1995. Sustainability and long-term dynamics of soil organic matter and nutrients under alternative management strategies. In: Bouma, J., Kuyvenhoven, A., Bouman, B.A.M., Luyten, J.C., Zandstra, H.G. (Eds.), Eco-Regional Approaches for Sustainable Land Use and Food Production. Kluwer Acad. Publ., Dordrecht, the Netherlands: 353-375.

Wiesner J.B., York H.F., 1964. Scientific American, 211(4): 27.

Wunder S. (2005). Payments for environmental services: Some nuts and bolts. CIFOR Occasional Paper, 42, Jakarta, Indonesia, $31 \mathrm{p}$.

Yunus M., 1997. Vers un monde sans pauvreté. Ed. Livre de Poche, Paris, 411 p. 